\title{
STDP encodes oscillation frequencies in the connections of recurrent networks of spiking neurons
}

\author{
Robert R Kerr ${ }^{1,2 *}$, Anthony N Burkitt ${ }^{1,2,4}$, Doreen A Thomas³, David B Grayden 1,2,4 \\ From Twenty First Annual Computational Neuroscience Meeting: CNS*2012 \\ Decatur, GA, USA. 21-26 July 2012
}

Spike-timing-dependent plasticity (STDP) is a learning rule that updates synaptic strengths based on the relative timing of pre- and post-synaptic spikes. Unlike ratebased Hebbian learning, STDP can potentially encode fast temporal correlations in neuronal activity, such as oscillations, in the functional structure of networks of neurons that have axonal and dendritic propagation delays. The motivation behind this study was to understand the different ways that spatiotemporal patterns can be learnt by the recurrent connections in a network of neurons with STDP present. This understanding is vital to uncovering the mechanisms by which basic learning and information processing tasks are performed throughout the brain. A specific example in which these mechanisms may contribute is in explaining how the brain can perceive the pitch of complex sounds up to $300 \mathrm{~Hz}$. This work employs and builds upon the analytical framework for learning with STDP used in a previous study [1].

In this study, the changes made by additive STDP to synaptic strengths in recurrent networks with axonal
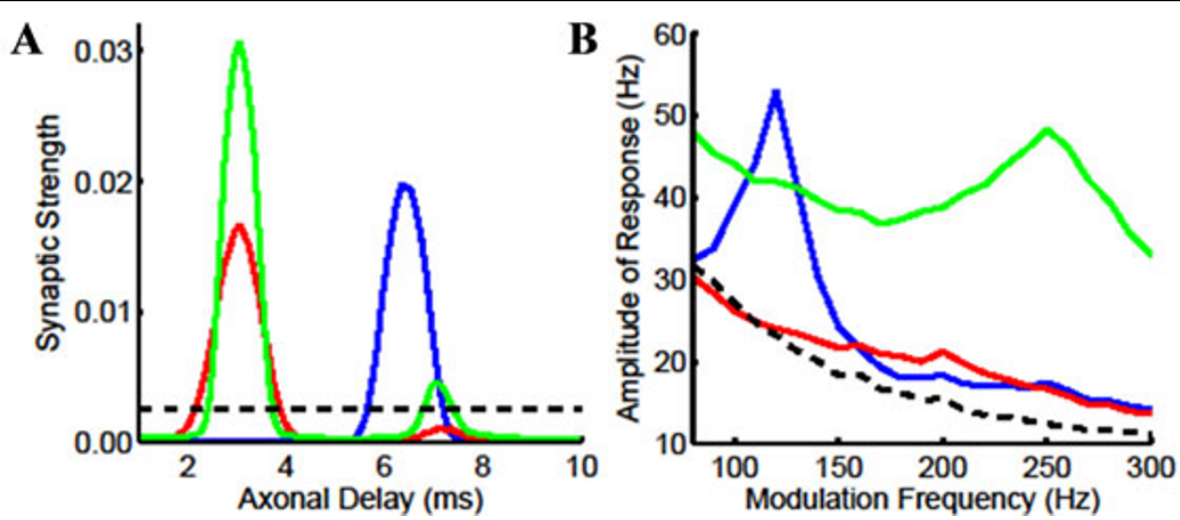

Figure 1 Results of simulations with networks of 10,000 LIF neurons. A: The mean synaptic weight with axonal delay of a network before learning (dashed: all weights initially set to 0.0025 with an even spread of delays over 1-10ms) and after learning for 20,000s with STDP (blue: 'medium' dynamics and $120 \mathrm{~Hz}$ inputs; red: 'medium' dynamics and $240 \mathrm{~Hz}$ inputs; and green: 'fast' dynamics and $240 \mathrm{~Hz}$ inputs). B: The frequencyresponse plot with the average maximum of the oscillatory response of the networks in A. Note: 'medium/fast' dynamics correspond to 10ms/ $5 \mathrm{~ms}$ membrane time constant, $0.5 \mathrm{~ms} / 0.1 \mathrm{~ms}$ synaptic rise time, and $1 \mathrm{~ms} / 0.5 \mathrm{~ms}$ synaptic decay time. These are realistic for neurons/synapses found in the auditory brainstem.

\footnotetext{
* Correspondence: r.kerr@pgrad.unimelb.edu.au

${ }^{1}$ NeuroEngineering Lab, Dept. Electrical \& Electronic Engineering, University of Melbourne, VIC 3010, Australia

Full list of author information is available at the end of the article
}

(c) 2012 Kerr et al; licensee BioMed Central Ltd. This is an Open Access article distributed under the terms of the Creative Commons 
delays receiving oscillatory inputs were investigated analytically with the Poisson neuron model and verified through simulations with leaky integrate-and-fire (LIF) neurons. Frequencies between $100-300 \mathrm{~Hz}$ were considered, which correspond to the modulation frequencies found in the auditory brainstem representing the fundamental frequency of different natural sounds. The analysis and simulations found that connections were selectively potentiated and depressed based on their axonal delay in such a way that the delays of the strong connections in the network "resonated" with the input frequency (Figure 1A). The trained networks were found to respond selectively to the frequency they were trained with (Figure 1B). Higher frequencies (e.g. $240 \mathrm{~Hz}$ as shown here) would always be learnt by the network, but in order to show a selective response after learning they needed faster neuronal and synaptic time constants (see details in Figure 1 caption).

\section{Acknowledgements}

Funding is acknowledged from the Australian Research Council (ARC Discovery Project \#DP1096699). The Bionics Institute acknowledges the support it receives from the Victorian Government through its Operational Infrastructure Support Program.

\section{Author details}

${ }^{1}$ NeuroEngineering Lab, Dept. Electrical \& Electronic Engineering, University of Melbourne, VIC 3010, Australia. ${ }^{2}$ Centre for Neural Engineering, University of Melbourne, VIC 3010, Australia. ${ }^{3}$ Dept. of Mechanical Engineering, University of Melbourne, VIC 3010, Australia. ${ }^{4}$ Bionics Institute, 384 Albert St., East Melbourne, VIC 3002, Australia.

Published: 16 July 2012

\section{Reference}

1. Gilson M, Burkitt AN, Grayden DB, Thomas DA, van Hemmen JL: Emergence of network structure due to spike-timing-dependent plasticity in recurrent neuronal networks IV: Structuring synaptic pathways among recurrent connections. Biological Cybernetics 2009, 101:427-444.

\section{doi:10.1186/1471-2202-13-S1-P130}

Cite this article as: Kerr et al:: STDP encodes oscillation frequencies in the connections of recurrent networks of spiking neurons. BMC Neuroscience 2012 13(Suppl 1):P130.

\section{Submit your next manuscript to BioMed Central and take full advantage of:}

- Convenient online submission

- Thorough peer review

- No space constraints or color figure charges

- Immediate publication on acceptance

- Inclusion in PubMed, CAS, Scopus and Google Scholar

- Research which is freely available for redistribution

Submit your manuscript at www.biomedcentral.com/submit 